\title{
Selection of optimal muscle set for 16-channel standing neuroprosthesis
}

\author{
Steven J. Gartman; ${ }^{*}$ Musa L. Audu, PhD; Robert F. Kirsch, PhD; Ronald J. Triolo, PhD \\ Department of Biomedical Engineering and Orthopedics, Case Western Reserve University, Cleveland, OH; Motion \\ Study Laboratory, Louis Stokes Cleveland Department of Veterans Affairs Medical Center, Cleveland, OH
}

\begin{abstract}
The Case Western Reserve University/Department of Veterans Affairs 8-channel lower-limb neuroprosthesis can restore standing to selected individuals with paraplegia by application of functional electrical stimulation. The second generation of this system will include 16 channels of stimulation and a closed-loop control scheme to provide automatic postural corrections. This study used a musculoskeletal model of the legs and trunk to determine which muscles to target with the new system in order to maximize the range of postures that can be statically maintained, which should increase the system's ability to provide adequate support to maintain standing when the user's posture moves away from a neutral stance, either by an external disturbance or a volitional change in posture by the user. The results show that the prime muscle targets should be the medial gastrocnemius, tibialis anterior, vastus lateralis, semimembranosus, gluteus maximus, gluteus medius, adductor magnus, and erector spinae. This set of 16 muscles supports $42 \%$ of the standing postures that are attainable by the nondisabled model. Coactivation of the lateral gastrocnemius and peroneus longus with the medial gastrocnemius and of the peroneus tertius with the tibialis anterior increased the percentage of feasible postures to $71 \%$.
\end{abstract}

Key words: biomechanical modeling, functional electrical stimulation, lower limb, muscle selection, musculoskeletal model, neuroprosthesis, optimization, rehabilitation, spinal cord injury, standing balance.

\section{INTRODUCTION}

Approximately 250,000 individuals in the United States have spinal cord injury (SCI) [1]. Of that population,
27.5 percent have complete paraplegia, which is the loss of all motor and sensory function below the level of injury [2], resulting in the loss of the ability to stand or walk.

One course of treatment for these individuals is the use of functional electrical stimulation (FES) to restore some abilities and rebuild muscle mass. One particular system, the Case Western Reserve University/Department of Veterans Affairs (CWRU/VA) standing neuroprosthesis, uses an implanted 8-channel stimulator to bilaterally activate the erector spinae, gluteus maximus, semimembranosus, and vastus lateralis muscles to extend the trunk, hips, and knees and prevent collapse. With the addition of external orthoses to stabilize the ankle and a means to maintain balance, such as upper-limb effort applied to a walker, users of the system can transition from a sitting position to a standing position and remain standing for clinically relevant amounts of time, averaging more than 10 minutes [3-5].

Two primary limitations to the current system exist. First, the system has a limited number of channels and therefore only a fraction of the total number of muscles in

\footnotetext{
Abbreviations: 3-D = three-dimensional, CWRU/VA = Case Western Reserve University/Department of Veterans Affairs, DOF = degree of freedom, FES = functional electrical stimulation, $\mathrm{SCI}=$ spinal cord injury, $\mathrm{SQP}=$ sequential quadratric programming.

* Address all correspondence to Steven J. Gartman, Motion Study Laboratory, 151A(W), Cleveland VA Medical Center, Cleveland, OH 44106; 216-791-3800, ext 3804; fax: 216231-3433. Email: steven.gartman@case.edu

DOI: 10.1682/JRRD.2007.10.0164
} 
the legs and trunk can be stimulated. This means that external orthoses are needed to stabilize the ankles since no muscles below the knee are recruited. Second, the system uses an open-loop control scheme and the stimulation amplitude is all-or-nothing. These factors lead to muscle fatigue and mean that the user must at times use their arms (via an assistive device such as a walker) to augment support.

Work is underway to develop the second generation of the system that will address these limitations. The new system will have 16 channels of stimulation and a feedback controller that will automatically adjust stimulation levels to provide three-dimensional (3-D) control of balance and posture. These changes should reduce the upper-body effort required to stand, extend standing times, and eliminate the need for external ankle orthoses.

The muscles selected for the existing 8-channel system were based on estimates of available muscle strength and surgical accessibility. This study used a modeling approach to offer a theoretical basis for the selection of an optimal muscle set for the new system before involving human subjects. We used a 3-D musculoskeletal model of bipedal stance representing the legs and trunk of an average adult male to explore the underlying mechanics and maximize the benefits of a 16-channel neuroprosthesis by selecting the "best" muscles for implantation. Specifically, the objective of this study was to optimize the muscle set such that the range of feasible postures in both the coronal and sagittal planes was maximized. This study extends work done in an earlier study [6] that focused on postures limited to the sagittal plane by testing the model for a range of postures in both the sagittal and coronal planes.

\section{METHODS}

\section{Model Description}

A static musculoskeletal model developed with SIMM software (version 4.1.1., MusculoGraphics, Inc; Santa Rosa, California) that had 15 degrees of freedom (DOF) and 40 muscles was used in this study to determine whether a set of fixed postures could be maintained given a particular set of active (stimulated) muscles (Figure 1). The articulations in the model were limited to the legs and trunk, with the head, arms, and trunk treated as a lumped mass. The trunk movement was also simplified such that the spine was treated as a rigid bar attached

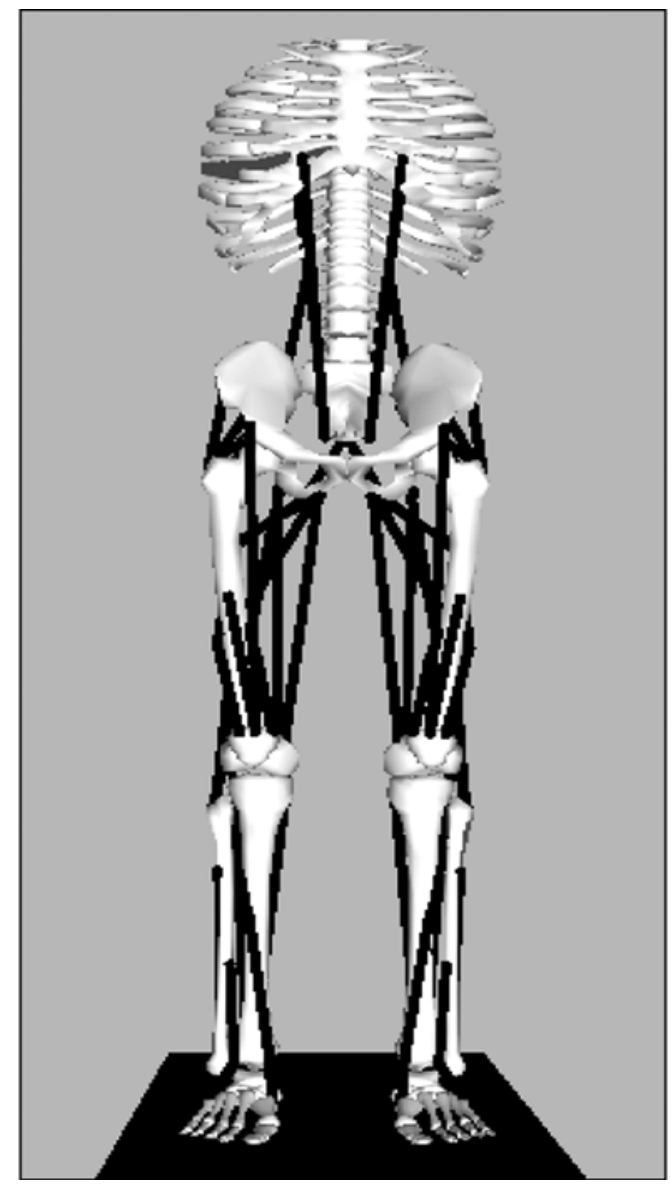

Figure 1.

Depiction of SIMM model. Degrees of freedom allowed in model were ankle eversion/inversion and flexion/extension; knee flexion/ extension; hip adduction/abduction, flexion/extension, and rotation; and trunk pitch, roll, and yaw. Model included 40 muscles: medial and lateral gastrocnemius, soleus, tibialis anterior, peroneals (brevis, longus, and tertius), vasti (medius, intermedius, and lateralis), rectus femoris, semimembranosus, biceps femoris (long and short head), adductor magnus, gluteus maximus, gluteus medius, erector spinae, quadratus lumborum, and rectus abdominis.

to the pelvis by a single ball joint [7]. The model was used to estimate two primary sets of information: (1) the moments required at each DOF to keep a given posture static and (2) the muscle activations required to generate those moments.

\section{Determining Required Moments}

Simulations performed with the model were first used to determine the joint moments required to maintain a given posture statically. Since the model contained a closed chain (both feet were attached to the ground, creating a 
closed chain bounded by the legs, ground, and pelvis), only 9 of the 15 DOFs were independent -6 in the legs and 3 at the ball joint between the trunk and the pelvis. This means that a posture can be completely controlled if six DOFs in the legs are actuated, leaving the remaining six as unactuated, floating joints. A previous study concluded that these six DOFs are best allocated to five DOFs that act primarily in the sagittal plane and one DOF that acts primarily in the coronal plane [8]. In the present study, the actuated joints in the sagittal plane were chosen to be left and right ankle dorsiflexion/plantar flexion, left and right knee flexion/extension, and right hip flexion/ extension. The actuated joint in the coronal plane was right hip adduction/abduction. Initial tests showed that this set of actuated DOFs required large moments at both hip DOFs as well as asymmetric distribution of moments, so the required moments for left hip flexion/extension and adduction/abduction were also calculated. Including these two additional DOFs in the analyses made the estimation of joint moments underconstrained. Therefore, these moments were calculated using a sequential quadratric programming (SQP) optimization routine [9] such that the sum of the squared joint moments for all DOFs was minimized. The remaining four DOFs (right and left ankle inversion and right and left hip rotation) were not actuated, so only passive moments (those created by ligaments and other passive structures, as described in Amankwah et al. [10]) were required at the joint.

\section{Determining Muscle Activations}

We determined the muscle activations required to generate the moments calculated previously using an SQP optimization routine [9] such that the overall muscle stress was minimized. Muscle activations ranged from zero to one, with a force output that was directly proportional to the activation level [11]. Coactivation of multiple muscles by a single channel was simulated by adding an optimization constraint that required the activation levels of all muscles in the group to be equal. Individual portions of the three large hip muscles (gluteus maximus, gluteus medius, and adductor magnus) could be individually activated since these muscles were divided into three separate sections in the model, although normally the whole muscle was assumed to be at the same activation level. If the specified muscle set could not generate sufficient moments to maintain the static posture, then the posture was deemed infeasible.

\section{Model Assumptions}

1. A single model using parameters based on averages from the adult male population [12-14] can provide meaningful insights into the mechanical properties of quiet standing for people with a range of anatomical parameters.

2. Muscles can be selectively stimulated; i.e., the whole muscle can be recruited without coactivation of adjacent muscles [15].

3. Since only static postures were examined, a static muscle model can be used with a force output that is directly proportional to the activation level [11].

4. Multiple muscles can be coactivated when desired via strategically located electrodes, and large muscles can be fully recruited with a single channel.

5. The effects of SCI on the force output of muscles can be represented by reducing the maximum isometric force of each muscle to 50 percent of the nondisabled maximum force.

6. The feet were shoulder-width apart, and sufficient friction with the ground to prevent moment of the feet was present.

7. FES-mediated postural control will be adequate if muscle forces are selected to minimize the total moments required to maintain the posture and if the muscle forces are distributed such that the total muscle stress is minimized.

8. Muscle actions will not cause excessive movement for ankle eversion/inversion, hip rotation, or trunk roll (twisting). Passive moments and friction between the feet and the ground should be sufficient to ensure this for hip rotation and trunk roll, although external orthoses may be required to ensure this for the subtalar joint (ankle eversion/inversion).

\section{Selection of Base Muscle Set}

A base set of 16 muscles was selected for the simulated FES standing system by computing the contributions of all muscles to joint moments in the sagittal and coronal planes for a wide range of standing postures (i.e., combinations of joint angles that produce useful standing configurations). Each posture was generated by specifying the position of six DOFs in the legs and each of the three DOFs for the lumbosacral joint. The positions of the remaining DOFs were calculated such that the closed chain between the feet was not broken. The specified DOFs were right and left ankle dorsiflexion, right and left knee flexion, one hip adduction angle, and one hip 
flexion angle. Note that the choice of which joint angles to specify is arbitrary, so we chose to specify the two hip DOFs as left hip flexion and adduction when the center of mass was on the left side of the center line between the feet and as right hip adduction and flexion when the center of mass was on the right side, thus ensuring symmetry between postures leaning to the left or to the right.

All 40 muscles were independently evaluated for inclusion in the base set of 16 FES muscles by the magnitude of their relative contributions to the required moments at each of the DOFs over a subset of postures limited to the sagittal and coronal planes. Hip musculature was tested using coronal plane postures. These postures were generated by varying the hip adduction angle from $-10^{\circ}$ to $+10^{\circ}$ (where the negative indicates abduction) in increments of $0.25^{\circ}$ while keeping both knees and ankles locked in their neutral positions $\left(0^{\circ}\right)$. Postures with hip adduction angles beyond $\pm 10^{\circ}$ were infeasible using the full muscle set and were excluded. The hips were maintained in slight flexion $\left(\sim 4^{\circ}\right)$ in order to bias the system toward requiring hip extension moments. Ankle and knee musculature was tested using sagittal plane postures. These postures were generated by bilaterally varying ankle dorsiflexion from $-4^{\circ}$ to $10^{\circ}$ (where the negative indicates plantar flexion) in increments of $0.25^{\circ}$ while keeping both knees locked in their neutral posture $\left(0^{\circ}\right)$. Postures beyond $-4^{\circ}$ and $+10^{\circ}$ of dorsiflexion were excluded since they were infeasible using the full muscle set. Here also, the hips were maintained in slight flexion $\left(\sim 4^{\circ}\right)$. In both sagittal and coronal plane postures, trunk pitch, roll, and yaw were specified to be in their neutral positions $\left(0^{\circ}\right)$. The joint moments required to maintain the posture and the muscle activations necessary to generate these moments were then calculated for each posture.

The particular 16 muscles that generated the greatest moments over each of the DOFs were selected as a base muscle set. This should be the simplest set of 16 muscles that produces the greatest range of feasible postures.

\section{Selection of Optimal Muscle Set}

Variations of the base muscle set were tested to determine whether the range of feasible postures could be improved by coactivating groups of two or more muscles such that more muscles were active while maintaining the 16-channel limit. As noted previously, coactivation of multiple muscles was achieved in the model by requiring that the activation level for each muscle in the group be equal. The base muscle set and variations were tested using a posture space that included postures in both the sagittal and coronal planes. The postures were generated in the same manner as before, but now all the combinations of the various hip adduction angles and ankle dorsiflexion angles were tested. To reduce the number of postures to a manageable level, we increased the increment by which each of these angles was varied from $0.25^{\circ}$ to $1^{\circ}$. Once again, knee angles were locked in their neutral position $\left(0^{\circ}\right)$, and the hips were maintained in slight flexion. For the tests of knee extensors, the posture space was altered slightly such that the knees were bilaterally slightly flexed $\left(4^{\circ}\right)$. This was done to stress the knee extensors by moving the center of mass posteriorly, thereby increasing the number of postures where a knee extension moment was required. To further examine the need to increase the moment capacity of the knee extensors, we simulated fatigue of the extensors by setting their maximum strength to 25 percent of their normal strength under SCI conditions.

\section{RESULTS}

Simulations were performed in the sagittal and coronal planes using all 40 muscles to determine the subset of 16 muscles that would provide the largest range of feasible postures. The hip and trunk musculature was evaluated for various coronal plane postures spanning the range depicted in Figure 2(a). Simulations were conducted in which the hip flexion/extension and adduction/abduction were actuated on only one side of the body and in which they were bilaterally actuated. In both cases, we found that across all the various DOFs, the highest required moments for postures over this range were for hip adduction/abduction. When only one hip was actuated, the required adduction moments for that hip were in the range of $-43 \mathrm{~N} \cdot \mathrm{m}$ to $76 \mathrm{~N} \cdot \mathrm{m}$ (with negative values indicating required abduction moment). Since the hip- spanning muscles included in the model can generate at most 31 $\mathrm{N} \cdot \mathrm{m}$ for abduction and $50 \mathrm{~N} \cdot \mathrm{m}$ for adduction, only a small range of the tested postures was feasible under these conditions. The required adduction/abduction moments were reduced when the hips were bilaterally actuated to a range of $-24 \mathrm{~N} \cdot \mathrm{m}$ to $22 \mathrm{~N} \cdot \mathrm{m}$ for both hips. Model simulations indicated that the required abduction moments were produced almost entirely by gluteus medius and hip adduction moments were produced almost entirely by adductor 


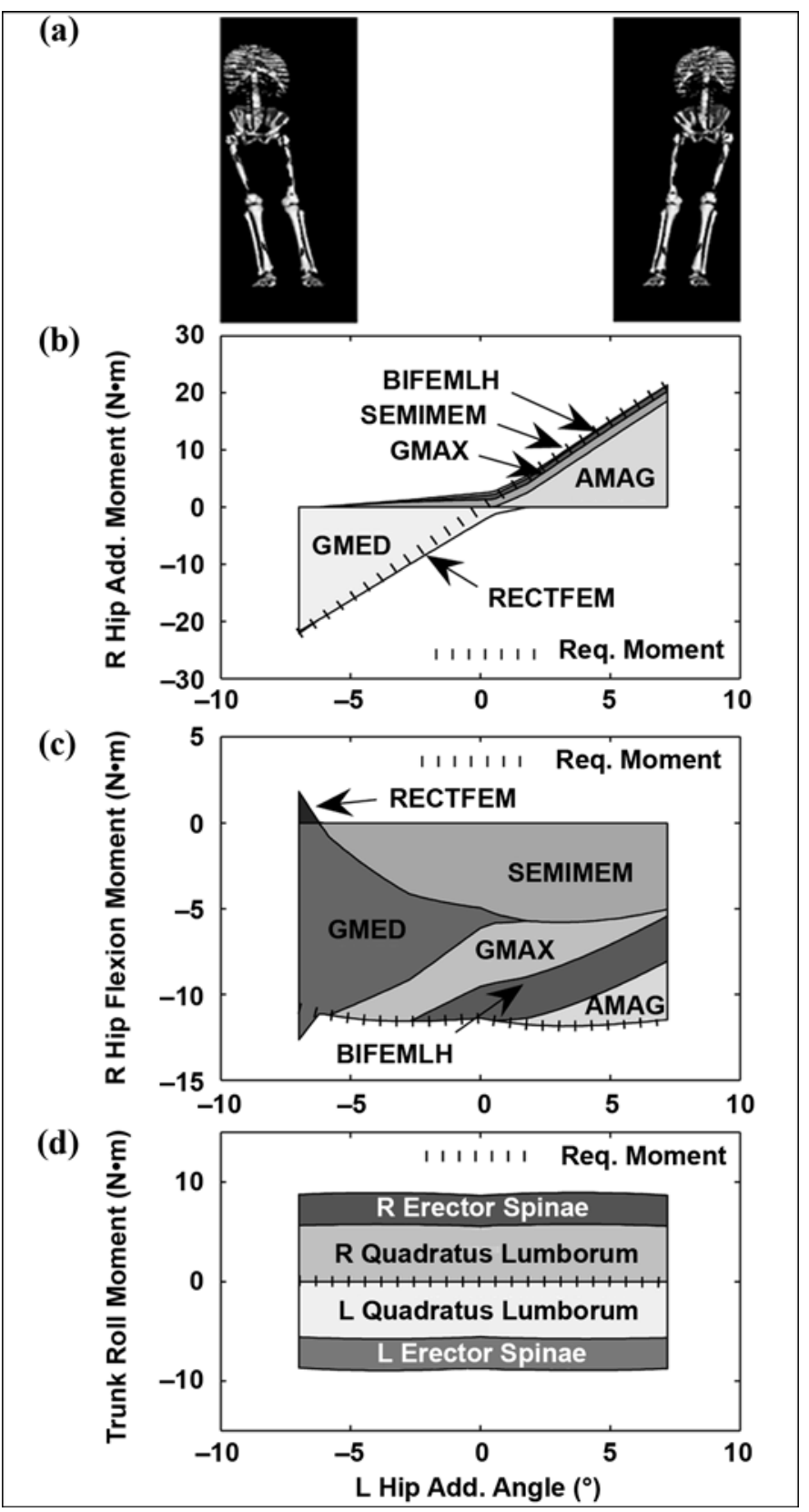

Figure 2.

Required moments for coronal plane postures and muscles that generate moments. (a) Posture space was generated by varying left hip adduction angle from $-7^{\circ}$ to $+7^{\circ}$, where negative value indications abduction. Hatched lines show moment required to statically maintain posture, and each shaded region represents moment contribution from particular muscle toward that required moment. Muscles that produced little moment may not have visible region but are still designated. Results are shown for three degrees of freedom: (b) right hip adduction, (c) right hip flexion, and (d) trunk roll. Add. = adduction, AMAG = adductor magnus, BIFEMLH = biceps femoris (long head), GMAX = gluteus maximus, GMED = gluteus medius, $\mathrm{L}=$ left, $\mathrm{R}$ = right, $\mathrm{RECTFEM} \mathrm{=} \mathrm{rectus} \mathrm{femo-}$ ris, Req. $=$ required, SEMIMEM $=$ semimembranosus. magnus. When the gluteus medius was activated to generate hip abduction moments, it also produced excessive hip extension moments that required rectus femoris-mediated hip flexion to maintain equilibrium. Otherwise, the semimembranosus produced the bulk of the required hip extension moments (Figure 2(c)). In the neutral postures, where the hip adduction angle was near $0^{\circ}$, the gluteus maximus provided roughly 30 percent of the required hip extension moment. At the lumbosacral joint, essentially no moment was required to maintain trunk roll. Both the quadratus lumborum and erector spinae were bilaterally active in order to satisfy the moment requirements for trunk pitch, but the trunk roll moments of these bilateral pairs canceled each other such that the net moment generated was equal to the required value of zero (Figure 2(d)).

Sagittal plane postures were used to test ankle, knee, and trunk musculature. Figure 3(a) shows a depiction of the model at both extremes of the tested posture space. In forward-leaning postures, the medial gastrocnemius generated most of the required plantar flexion moment, followed by the soleus and lateral gastrocnemius (Figure 3(b)). Both of the gastrocnemii served a secondary function of providing knee flexion moment to keep the knee from hyperextending (Figure 3(c)). The muscles included in the model could not support postures with ankle angles greater than $7^{\circ}$ because the gastrocnemii became fully activated at this point and could not generate any more knee flexion moment. In backward-leaning postures, the vasti were active in roughly equal amounts to provide knee extension moments and the tibialis anterior and peroneus tertius were active to provide a dorsiflexion moment. Little or no activation of the peroneal muscles was noted.

Using the data shown in Figures 2 and 3, we selected the set of 16 muscles that provided the greatest moment for each of the DOFs to be used as a base muscle set. These muscles were medial gastrocnemius for plantar flexion, tibialis anterior for dorsiflexion, vastus intermedius for knee extension, semimembranosus for knee flexion, adductor magnus for hip adduction, gluteus medius for hip abduction, gluteus maximus for hip extension, and erector spinae for trunk pitch and roll. Although the quadratus lumborum does generate more moment than the erector spinae for trunk roll, the moment required for trunk pitch is typically greater and the erector spinae generates more moment for this DOF (Figure 3(d)).

This base set of 16 muscles was then used in simulations to evaluate its performance for postures shifted in both the coronal and sagittal planes. Figure 4 shows a 


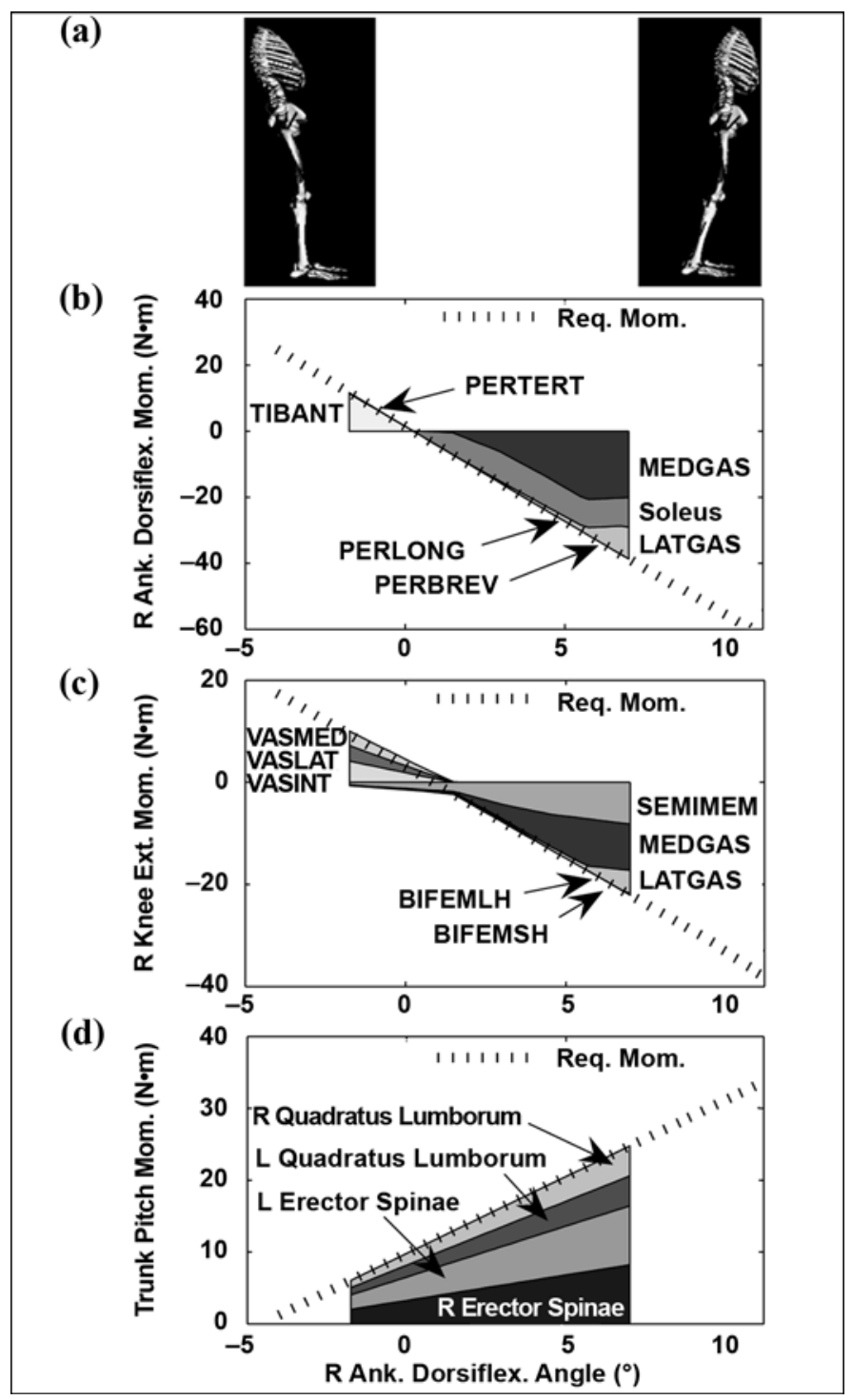

Figure 3.

Required moments for sagittal plane postures and muscles that generate moments. (a) Posture space was generated by bilaterally varying ankle angle from $-4^{\circ}$ to $+10^{\circ}$, where negative value indicates plantar flexion. Hatched lines show moment required to statically maintain posture, and each shaded region represents moment contribution from particular muscle toward that required moment. Muscles that produced little moment may not have visible region but are still designated. Results are shown for three degrees of freedom: (b) right ankle dorsiflexion, (c) right knee extension, and (d) trunk pitch. Ank. = ankle, BIFEMLH = biceps femoris (long head), BIFEMSH = biceps femoris (short head), Dorsiflex. = dorsiflexion, Ext. = extension, $\mathrm{L}=$ left, LATGAS = lateral gastrocnemius, MEDGAS = medial gastrocnemius, Mom. = moment, PERBREV = peroneus brevis, PERLONG = peroneus longus, PERTERT = peroneus tertius, $\mathrm{R}$ = right, Req. = required, SEMIMEM = semimembranosus, TIBANT $=$ tibialis anterior, VASINT $=$ vastus intermedius, VASLAT = vastus lateralis, VASMED = vastus medialis. graphical representation of the tested posture space, where the position in the coronal plane is identified by the left hip adduction angle (on the $x$-axis) and the position in the sagittal plane is identified by the ankle angles (on the $y$-axis). The grid of points on the plot represents the specific postures tested. The area enclosed by the dotted line shows the postures that were feasible using the nondisabled (40 muscle) model, while the shaded area shows the range of postures feasible using the 16-muscle base set. Overall, the base muscle set was found to support 42 percent of the postures that were feasible when all 40 muscles in the model were included. These postures were roughly centered about the nominal upright posture.

Stimulation of multiple adjacent muscles with one electrode was simulated by requiring identical activation levels for each of the grouped muscles. This can be achieved in the neuroprosthesis by placement of the electrodes near motor points of adjacent muscles [16], branching electrodes [17], or placing electrodes near nerves that innervate multiple muscles [18]. To test whether the results from the base muscle set could be improved by

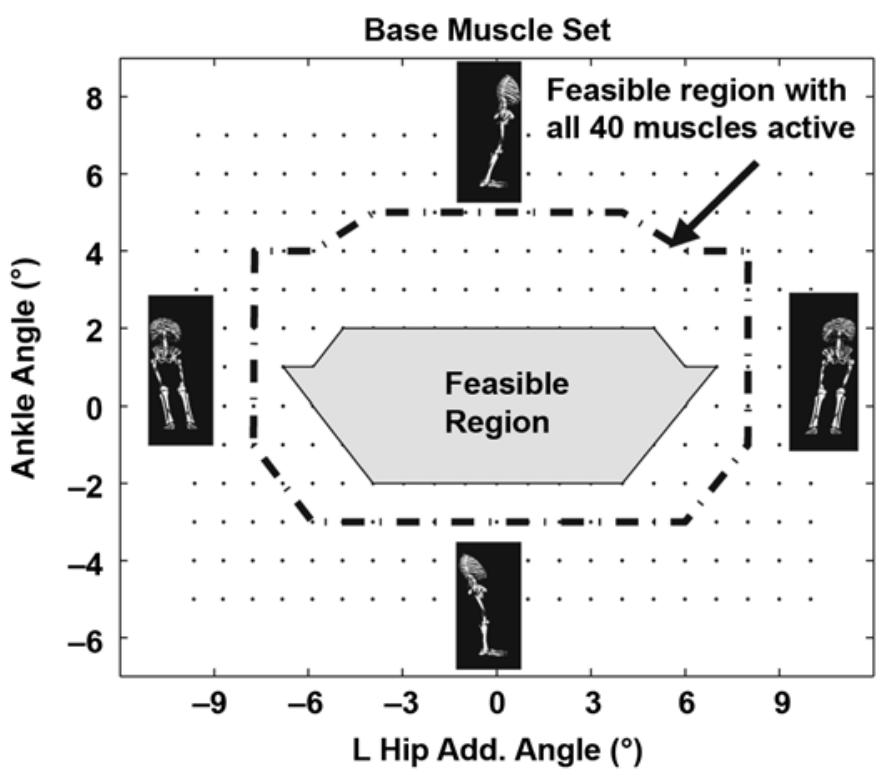

Figure 4.

Feasible posture space using base set of muscles. Variety of standing postures were created by varying left hip adduction angle (for coronal plane variations) and ankle angles bilaterally (for sagittal plane variations) while knees were fully extended and hip was maintained in slight flexion. Each posture is represented on plot by point. Shaded area shows posture space that is feasible given specified muscle set (in this case base muscle set), and dotted line shows posture space that is feasible with all 40 muscles active. Add. = adduction, $\mathrm{L}=$ left. 
stimulating multiple muscles using a single channel, we tested several specific muscle combinations at both the ankle and the knee. The combinations that were tested are presented in Figure 5(a) (for ankle muscle combinations) and Figure 6(a) (for knee muscle combinations).

The base muscle set has two channels designated for each ankle-one for plantar flexion and one for dorsiflexion. Each of the major plantar flexors (medial and lateral gastrocnemii and soleus) was activated individually and then in combination. Individually, the medial gastrocnemius allowed for the greatest number of feasible postures (Figure 5(b)). These results were improved by coactivating both the medial and lateral gastrocnemius, since together the muscles generate more knee flexion moment (Figure 5(c)). Coactivating the soleus with the gastrocnemii actually reduced the number of feasible postures. This is because strong activation of the gastrocnemii to generate required knee flexion moments was then linked to strong activation of the soleus, which generated excessive ankle plantar flexion moment that negatively affected overall performance.

The minor plantar flexors (peroneus longus and brevis) were then coactivated with the gastrocnemii, first individually and then together. Individually, both peroneals resulted in a greater number of feasible postures, with the peroneus longus slightly better than the peroneus brevis. Coactivating both peroneals with both gastrocnemii did not improve performance beyond the combination of just the peroneus longus with the gastrocnemii.

Peroneus tertius, the only other ankle dorsiflexor in the model, was coactivated with the tibialis anterior while using the best result with the plantar flexors (both gastrocnemii and peroneus longus). This combination also resulted in a slight increase in the number of feasible postures and was the best combination of ankle muscles that was tested (Figure 5(d)).

A variety of both individual knee muscles and knee muscle combinations were tested (Figure 6(a)). Under normal SCI conditions, any one of the knee extensors was sufficient to generate the moment required to keep the knee extended. The maximum required knee extension moment for the tested posture space was $13 \mathrm{~N} \cdot \mathrm{m}$, while the vastus medius, the weakest of the knee extensors, could generate $15 \mathrm{~N} \cdot \mathrm{m}$ across all the tested postures. In order to discriminate between the extensors, we simulated fatigue by setting the maximum force output of the extensors to 25 percent of their normal SCI strength. While the process of determining the base set of muscles showed

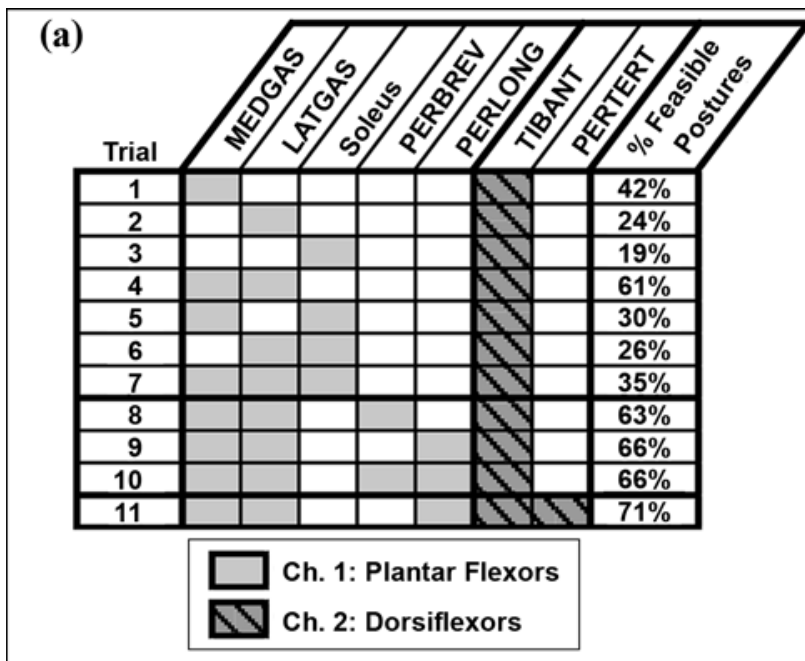

(b)

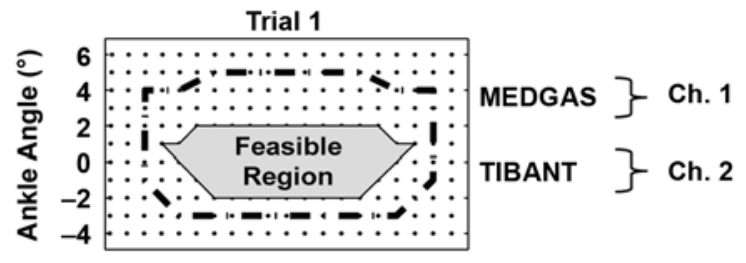

(c)

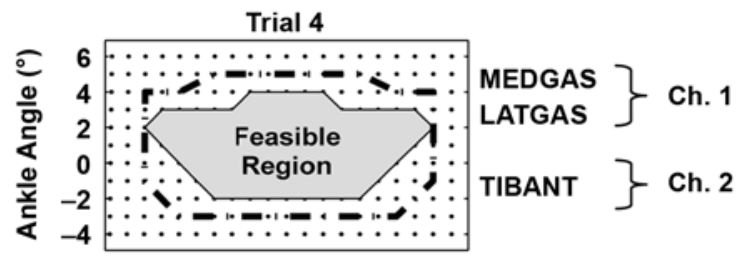

(d)

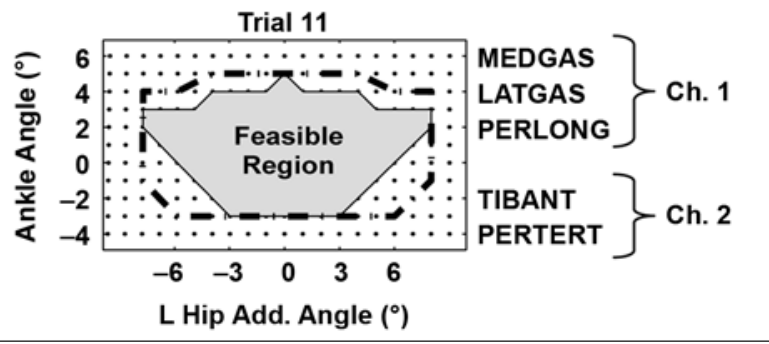

Figure 5.

Ankle muscle combinations. (a) Various muscle combinations were tested for muscles acting about ankles. Shaded portions indicate that muscle is active in particular trial, and multiple shaded cells indicate that muscles are coactivated at equal levels. Number of feasible postures is presented as percentage of number of postures feasible when all 40 muscles are active. (b-d) Feasible posture space is shown for selected trials. Each point represents tested posture. Shaded region shows postures that are feasible using given muscle combinations, while dotted border encompasses postures that are feasible when all 40 muscles are active. Add. = adduction, Ch. = channel, $\mathrm{L}=$ left, LATGAS = lateral gastrocnemius, MEDGAS = medial gastrocnemius, PERBREV = peroneus brevis, $\mathrm{PERLONG}=$ peroneus longus, PERTERT $=$ peroneus tertius, TIBANT $=$ tibialis anterior. 


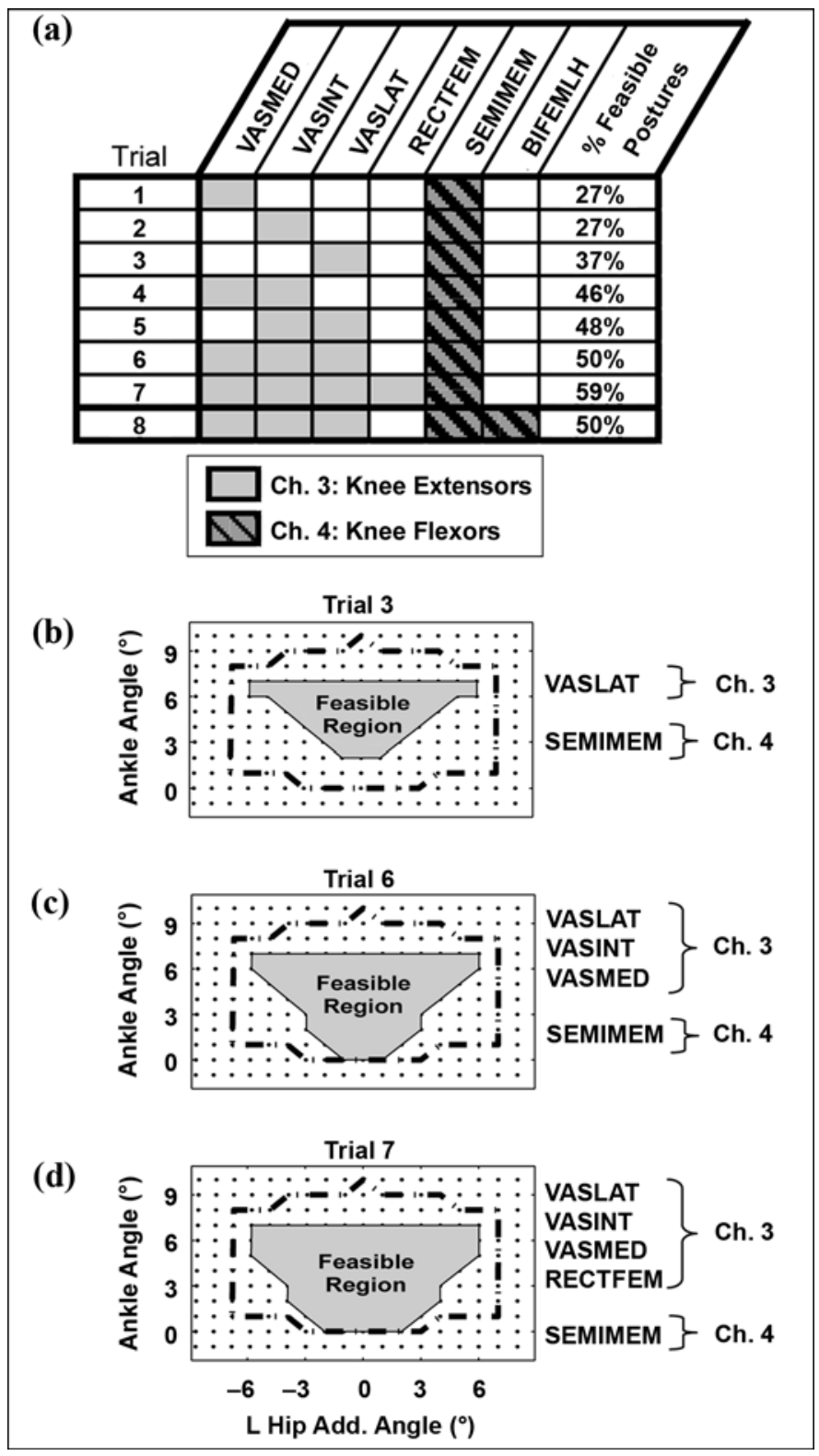

Figure 6.

Knee muscle combinations. (a) Various muscle combinations were tested for muscles acting about knees. Shaded portions indicate that muscle is active in particular trial, and multiple shaded cells indicate that muscles were coactivated at equal levels. Number of feasible postures is presented as percentage of number of postures feasible when all 40 muscles are active. (b-d) Feasible space is shown for selected trials. Each point represents tested posture. Shaded region shows postures that are feasible using the given muscle combinations, while dotted border encompasses postures that are feasible when all 40 muscles are active. Add. = adduction, BIFEMLH = biceps femoris (long head), Ch. = channel, $\mathrm{L}=$ left, RECTFEM = rectus femoris, SEMIMEM = semimembranosus, VASINT $=$ vastus intermedius, VASLAT $=$ vastus lateralis, VASMED = vastus medius. that vastus intermedius was the most efficient muscle in generating knee extension moment, the simulations with fatigue indicated that the vastus lateralis (which is larger and produces more knee extension moment) would support a wider range of postures (Figure 6(b)). Furthermore, combining the vastus intermedius with either the vastus lateralis or vastus medius substantially increased the range of feasible postures. Simultaneous activation of all three vasti (Figure 6(c)) was only marginally better than the two-vasti combinations, but adding the rectus femoris to all three vasti increased the number of feasible postures by an additional 9 percent (Figure 6(d)).

Since the long head of the biceps femoris and semimembranosus are both innervated by the tibial component of the sciatic nerve, coactivation of these muscles was also tested. This muscle combination gave no benefit over stimulating the semimembranosus alone.

Situations in which individual portions of the three large hip muscles (gluteus maximus, gluteus medius, and adductor magnus) might be activated separately from one another were simulated (Figure 7). Each of these three muscles was divided into two portions. The gluteus maximus and adductor magnus were divided into a superior and inferior portion, and the gluteus medius was divided into an anterior and posterior portion. The ankle and knee muscles included were those that gave the best results in the ankle and knee tests (i.e., both gastrocnemii and peroneus longus coactivated, tibialis anterior and peroneus tertius coactivated, and semimembranosus and all three vasti coactivated). The posture space that was tested was the same as in the ankle muscle selection.

Either of the two portions of the gluteus maximus yielded the same range of feasible postures as when the whole muscle was activated (Figure 7(b)). This was not true in the case of the adductor magnus and the gluteus medius, however. Activating the inferior portion of the adductor magnus as opposed to the superior portion caused a decrease in the range of feasible postures in the coronal plane because of the decrease in available hip adduction moment (Figure 7(c)). Activating the superior portion alone yielded the same range of feasible postures as when the whole muscle was active, showing that the superior portion is able to generate sufficient adduction moments and that the range is limited by some other factor. Stimulating only a portion of the gluteus medius also decreased the range of feasible postures in the coronal plane because of the decrease in available hip abduction moment (Figure 7(d)). Unlike the adductor magnus, 


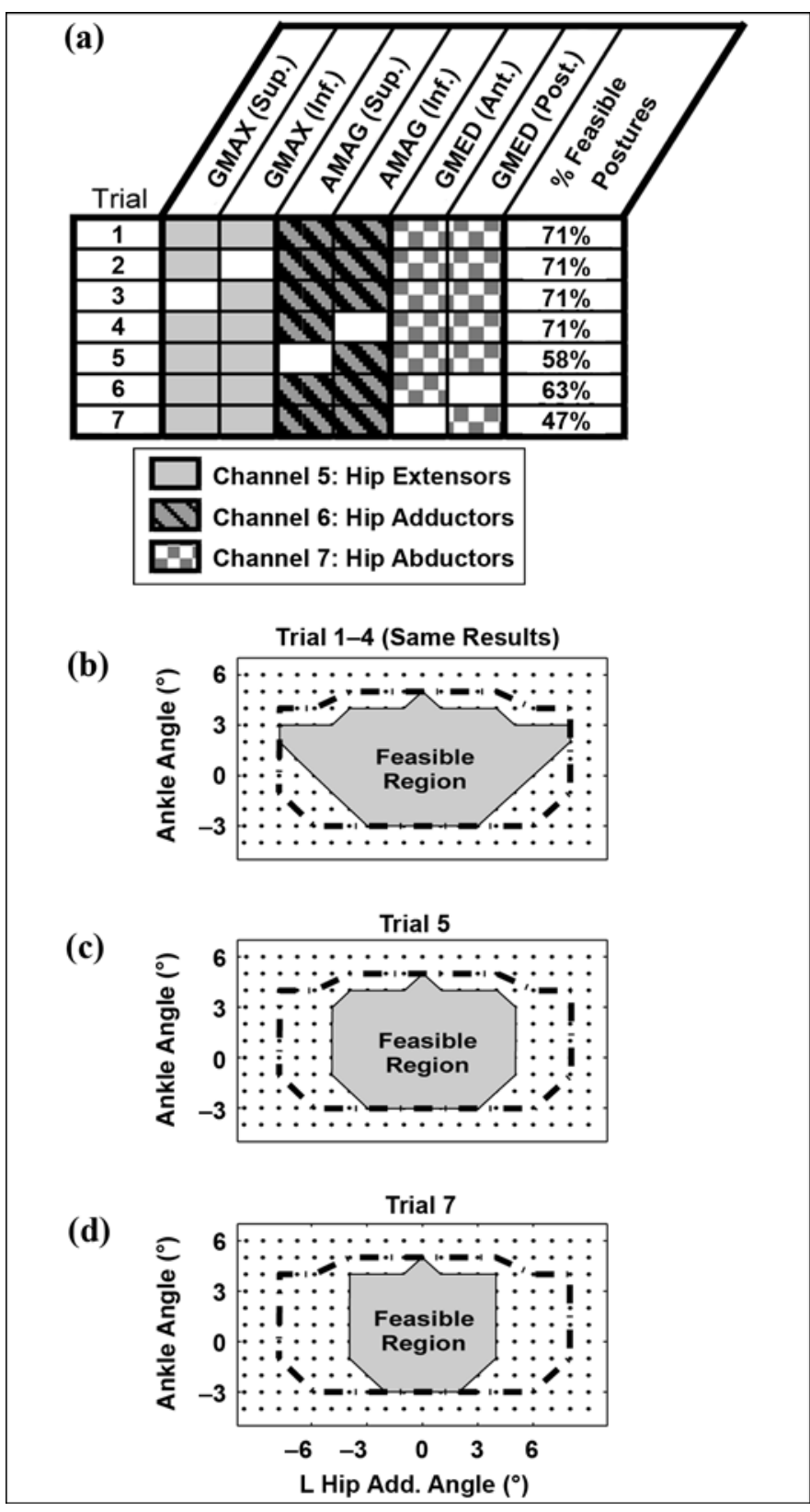

Figure 7.

Hip muscle combinations. (a) Various muscle combinations were tested for muscles acting about hips. Shaded portions indicate that muscle is active in particular trial, and multiple shaded cells indicate that muscles were coactivated at equal levels. Number of feasible postures is presented as percentage of number of postures feasible when all 40 muscles are active. (b-d) Feasible space is shown for selected trials. Each point represents tested posture. Shaded region shows postures that are feasible using given muscle combination, while dotted border encompasses postures that are feasible when all 40 muscles are activated. Add. = adduction, AMAG = adductor magnus, Ant. = anterior, GMAX = gluteus maximus, GMED = gluteus medius, Inf. = inferior, $\mathrm{L}=$ left, Post. = posterior, Sup. = superior. neither portion of the gluteus medius was sufficient to generate the same range as when the whole muscle was activated.

\section{DISCUSSION}

The number of electrodes that can be implanted in a functional neuromuscular stimulation system is necessarily limited by both technological and surgical constraints. Systems that provide the most function with the fewest stimulation channels are the ideal. The existing 8-channel CWRU/VA system allowed users to stand, which was useful in reaching activities and transferring in and out of a wheelchair. The system also allowed for exercise of the leg muscles, which was useful in building muscle mass, reducing the risk of pressure sores, improving circulation, and reducing muscle spasticity [19-20]. It is reasonable to ask what additional benefits a new 16-channel system will confer to users and how best to implement the system in order to maximize these benefits. This study helps address both of these issues by determining the increased range of standing postures (and hence the increased functionality) that would be made available by the advanced 16-channel system and by evaluating which particular set of muscles would provide the most additional functionality. Maximizing the range of standing postures that are feasible statically should increase the system's ability to provide adequate support to maintain standing when the user's posture moves away from a neutral stance, either by an external disturbance or a volitional change in posture by the user. While the present study focuses only on static postures, the results should apply to situations in which the disturbances to posture are small or the changes in posture are slow, thus allowing any dynamic effects to be neglected.

The 16-channel system proposed here should include 2 channels allocated to each ankle-one for plantar flexion and one for dorsiflexion. Of the plantar flexors, the model shows that the medial gastrocnemius gave the greatest range of feasible postures when stimulated alone and that coactivation with the lateral gastrocnemius and peroneus brevis or longus improved the results. Targeting the soleus decreased performance. An earlier study had indicated the soleus as the first plantar flexor to target [6], although differences between activation of the soleus versus the combined medial and lateral gastrocnemii were minimal. The earlier study also focused on a wider 
range of postures, including ones that had greater knee flexion angles where muscle actions producing knee flexion would be highly undesirable. The current study focused on more postures that were nearly fully erect, and a number of these postures required knee flexion moments (as would be provided by the gastrocnemii) to prevent knee hyperextension. The passive resistance to knee hyperextension is commonly used in current FES standing systems, so the issue of whether to include muscles such as the gastrocnemii in future FES systems to prevent such hyperextension is an open question. A clear conclusion, however, is that the results of the earlier study [6] and our current study indicate that model-based approaches can be used to optimize a number of different system properties.

The tibialis anterior should be the primary dorsiflexor target. Coactivation of the peroneus tertius improved the range of feasible postures. In addition, activation of the peroneus tertius could help prevent ankle inversion caused by the tibialis anterior. These results are consistent with the sagittal plane-only results described previously [6].

We found that any one of the vasti was individually strong enough to generate sufficient knee extension moments for the postures that were tested, even under SCI conditions. By simulating significant fatigue, we determined that the vastus lateralis should be the primary target, although performance was continuously improved as additional knee extensor muscles were added (coactivated by a single electrode). Although including the rectus femoris with the vasti also improved the range of feasible postures, its hip flexion action could also lead to an increased rate of fatigue of the hip extensors and ultimately a decrease in performance.

\section{CONCLUSIONS}

The main new contribution of this project was that it extended our model-based analysis of standing performance to postures in the coronal plane as well as the sagittal plane. In a previous study, the adductor magnus was included on the assumption that it would be used in coronal plane posture shifts [6]. This study shows that the adductor magnus is very important in providing hip adduction moments in sideways leaning postures and together with the gluteus medius helps stabilize postures in the coronal plane. Because of the closed-chain nature of standing, it is theoretically possible to control coronal plane posture via abduction and adduction on just one side of the body or via either abduction or adduction on both sides. However, our results suggest that controlling both abduction and adduction bilaterally will provide better performance because of the limited strength of the muscles.

The single best electrode combination suggested by this study consists bilaterally of the gastrocnemii coactivated with the peroneus longus (using a branched intramuscular electrode), the tibialis anterior coactivated with the peroneus tertius (via stimulation of the deep peroneal nerve), all of the vasti coactivated with the rectus femoris (via stimulation of the femoral nerve), and intramuscular electrodes in the semimembranosus, gluteus maximus, gluteus medius (with emphasis on the anterior portion), adductor magnus (with emphasis on the superior portion), and erector spinae. We focused on a very important, but relatively small subset of the infinite posture space that is possible, and different muscles may be more important in some of the untested postures. In addition, real-world systems come with many other considerations, such as individual user needs, desires, and functional limitations caused by the level of SCI, muscle spasticity, and muscle atrophy. It is promising that this study shows that a certain amount of flexibility exists in the 16-channel system when choosing muscles, in that many of the muscle combinations tested supported more than half of the postures that are feasible when all muscles are active. The specific results presented here, as well as the process that could be used for other scenarios, should be used to help guide and support decisions about which muscles to target for standing postures.

\section{ACKNOWLEDGMENTS}

This material was based on work supported by the National Institutes of Health, grant R01NS040547-03.

The authors have declared that no competing interests exist.

\section{REFERENCES}

1. Jackson AB, Dijkers M, Devivo MJ, Poczatek RB. A demographic profile of new traumatic spinal cord injuries: Change and stability over 30 years. Arch Phys Med Rehabil. 2004;85(11):1740-48. [PMID: 15520968] 
2. National Spinal Cord Injury Statistical Center. The 2006 annual statistical report for the model spinal cord injury care systems. Birmingham (AL): National Spinal Cord Injury Statistical Center. 2006. p. 124.

3. Triolo RJ, Bieri C, Uhlir J, Kobetic R, Scheiner A, Marsolais EB. Implanted Functional Neuromuscular Simulation systems for individuals with cervical spinal cord injuries: Clinical case report. Arch Phys Med Rehabil. 1996;77(11): 1119-28. [PMID: 8931521]

4. Davis JA Jr, Triolo RJ, Uhlir JP, Bhadra N, Lissy DA, Nandurkar S, Marsolais EB. Surgical technique for installing an eight-channel neuroprosthesis for standing. Clin Orthop Relat Res. 2001;(385):237-52. [PMID: 11302320]

5. Davis JA Jr, Triolo RJ, Uhlir J, Bieri C, Rohde L, Lissy D, Kukke S. Preliminary performance of a surgically implanted neuroprosthesis for standing and transfers-Where do we stand? J Rehabil Res Dev. 2001;38(6):609-17. [PMID: 11767968]

6. Heilman BP, Audu ML, Kirsch RF, Triolo RJ. Selection of an optimal muscle set for a 16-channel standing neuroprosthesis using a human musculoskeletal model. J Rehabil Res Dev. 2006;43(2):273-86. [PMID: 16847793]

7. Audu ML, Kirsch RF, Triolo RJ. A computational technique for determining the ground reaction forces in human bipedal stance. J Appl Biomech. 2003;19(4):361-71.

8. Kim JY, Popovic MR, Mills JK. Dynamic modeling and torque estimation of FES-assisted arm-free standing for paraplegics. IEEE Trans Neural Syst Rehabil Eng. 2006; 14(1):46-54. [PMID: 16562631]

9. Zhou JL, Tits AL, Lawrence CT. A FORTRAN code for solving constrained nonlinear (Minimax) optimization problems. College Park (MD): Electrical Engineering Department and Institute for Systems Research, University of Maryland; 1997. p. 1-44.

10. Amankwah K, Triolo RJ, Kirsch R. Effects of spinal cord injury on lower-limb passive joint moments revealed through a nonlinear viscoelastic model. J Rehabil Res Dev. 2004; 41(1):15-32. [PMID: 15273894]
11. Zajac FE. Muscle and tendon: Properties, models, scaling, and application to biomechanics and motor control. Crit Rev Biomed Eng. 1989;17(4):359-411. [PMID: 2676342]

12. Brand RA, Pedersen DR, Friederich JA. The sensitivity of muscle force predictions to changes in physiologic crosssectional area. J Biomech. 1986;19(8):589-96.

[PMID: 3771581]

13. Wickiewicz TL, Roy RR, Powell PL, Edgerton VR. Muscle architecture of the human lower limb. Clin Orthop Relat Res. 1983;(179):275-83. [PMID: 6617027]

14. Friederich JA, Brand RA. Muscle fiber architecture in the human lower limb. J Biomech. 1990;23(1):91-95. [PMID: 2307696]

15. Triolo RJ, Liu MQ, Kobetic R, Uhlir JP. Selectivity of intramuscular stimulating electrodes in the lower limbs. J Rehabil Res Dev. 2001;38(5):533-44. [PMID: 11732831]

16. Marsolais EB, Kobetic R. Functional electrical stimulation for walking in paraplegia. J Bone Joint Surg Am. 1987; 69(5):728-33. [PMID: 3496340]

17. Uhlir JP, Triolo RJ, Kobetic R. The use of selective electrical stimulation of the quadriceps to improve standing function in paraplegia. IEEE Trans Rehabil Eng. 2000;8(4):514-22. [PMID: 11204043$]$

18. Brindley GS, Polkey CE, Rushton DN. Electrical splinting of the knee in paraplegia. Paraplegia. 1979;16(4):428-37. [PMID: 311910]

19. Bogie KM, Triolo RJ. Effects of regular use of neuromuscular electrical stimulation on tissue health. J Rehabil Res Dev. 2003;40(6):469-75. [PMID: 15077659$]$

20. Betz R, Boden B, Triolo R, Mesgarzadeh M, Gardner E, Fife R. Effects of functional electrical stimulation on the joints of adolescents with spinal cord injury. Paraplegia. 1996;34(3): 127-36. [PMID: 8668353]

Submitted for publication December 10, 2007. Accepted in revised form April 7, 2008. 